\title{
Nonlinear Thermoelastic Materials with Viscosity, and Subject to Internal Constraints: A Classical Continuum Thermodynamics Approach
}

\author{
James Casey
}

Received: 30 September 2010 / Published online: 14 January 2011

(C) The Author(s) 2011. This article is published with open access at Springerlink.com

\begin{abstract}
A general approach to continuum thermodynamics that was advocated by R.S. Rivlin is carried out for thermoelastic materials which can also depend on strain rate. An entropy function is constructed (rather than assumed to exist). A method for treating thermomechanical internal constraints for such materials is also presented. In this method, the properties of a constrained material are inherited from those of a related equivalence class of unconstrained materials.
\end{abstract}

Keywords Continuum thermodynamics · Thermoelasticity · Viscosity · Internal constraints

Mathematics Subject Classification (2000) 74A15 - 74A20 - 74B20 - 76A02

\section{Introduction}

There is a vast literature on continuum thermodynamics, much of which is strongly influenced by the work of Coleman and Noll (see [1,2]), in which an entropy function is admitted as a primitive variable. Carlson [3] adopts this approach in deriving a linearized theory of thermoelasticity.

An alternative, more classical, approach to thermodynamics was proposed by Rivlin [4-6], whereby for any given class of materials, special processes are specified which, in conjunction with "Part 1" of the second law of thermodynamics, lead to the construction of an entropy function. ${ }^{1}$ Generally speaking, the more complicated the class of materials, the more difficult it is to establish the existence of an entropy function. The advantage, however,

\footnotetext{
${ }^{1}$ Rivlin's approach to the thermodynamics of deformable media has been applied in [7-11].

In Memory of Don Carlson.

J. Casey $(\bowtie)$

Department of Mechanical Engineering, University of California, Berkeley, 6125 Etcheverry Hall, Berkeley, CA 94720-1740, USA

e-mail: jcasey@me.berkeley.edu
} 
is that one is forced to be explicit about the conditions under which the entropy function can be constructed. In contrast, in the postulational approach of Coleman and Noll [1, 2], entropy is just assumed to exist and no indication is given of any process that would lead to its construction.

After some preliminaries in Sect. 2, a general class of thermoelastic materials with viscosity is considered in Sect. 3. As in classical thermodynamics, it is important to distinguish between equations of state for such materials and the remaining constitutive equations. In particular, $\varepsilon(X, t)$, the internal energy per unit mass at a particle $X$ of a body at time $t$, is uniquely determined by the value of Lagrangian strain $\mathbf{E}$ at $(X, t)$ and the value of the absolute temperature $\theta(X, t)$, as indicated in $(19)_{1}$. This is an equation of state, the state being uniquely specified by $(\mathbf{E}, \theta) .^{2}$ In contrast, as will become evident, only a portion of the symmetric Piola-Kirchhoff stress tensor $\mathbf{S}$ is determined by $(\mathbf{E}, \theta)$, and hence $(19)_{2}$ is not an equation of state. However, (25), which gives the value $\mathbf{S}^{e}$ of $\mathbf{S}$ that would be measured if $\mathbf{E}$ and $\theta$ were held fixed, is an equation of state, and $\mathbf{S}^{e}$, like $\varepsilon$, is a state function. "Part 1" of the second law of thermodynamics enables us to construct other state functions, in particular entropy $\eta$ and Helmholtz free energy $\psi$. Further, as shown in Sect. 3, for thermoelastic materials with viscosity, the equilibrium stress and entropy are obtainable from $\psi$ as a potential function (see $(40)_{1,2}$ ). The corresponding equilibrium value of Cauchy stress is given by (43).

In Sect. 4, strain-temperature internal constraints for thermoelastic materials with viscosity are considered, using a method introduced by Casey and Krishnaswamy [7]. In this approach, the constraint manifold is used to partition the class of unconstrained materials in Sect. 3 into disjoint equivalence classes. The materials in an equivalence class share certain properties on the constraint manifold. A unique constrained material is made to correspond to each equivalence class of unconstrained materials. The properties of a constrained material are inherited from those of the corresponding equivalence class of unconstrained materials.

A list of references to other treatments of internally constrained materials may be found in [7].

\section{Preliminaries}

Consider a deformable 3-dimensional continuum $\mathcal{B}$ moving in inertial space under the influence of forces and subject to heating. Let $\mathbf{x}$ denote the position vector of a particle $X \in \mathcal{B}$ in the body's current configuration, at time $t$. Let $\mathbf{X}$ be the position vector of $X$ in a fixed occupiable reference configuration. Also, let $\theta(>0)$ be the absolute temperature at $X$ in the

\footnotetext{
${ }^{2}$ In the spirit of classical thermodynamics, a state (or equilibrium state) of the particle $X$ (in practice, a small homogeneous body) of thermoelastic material with viscosity may be defined as the collection of all physical properties that are uniquely determined by fixing the values of $\mathbf{E}$ and $\theta$. Since all of the other thermodynamic properties (or other "parameters of state") are fixed once the values of $\mathbf{E}$ and $\theta$ are given, there is a one-toone correspondence between the set of states and the 7-dimensional space to which the point $(\mathbf{E}, \theta)$ belongs. Thus, a state of the material point $X$ may be represented by a point in the strain-temperature space. Clearly, any topological image of the latter space could also be used to represent the states, corresponding to different choices of independent variables. For a general process, $(\mathbf{E}, \theta)$ becomes a parameterized function of time, producing a curve in strain-temperature space; some variables, such as stress, will depend on the strain rate $\dot{\mathbf{E}}$, while state functions are now determined by the instantaneous values of $\mathbf{E}$ and $\theta$. For equilibrium processes, the state remains fixed. It is always possible to hold $X$ in equilibrium in any desired state, but in general non-equilibrium processes, $X$ must go through a 1-parameter family of states and cannot be expected to be in equilibrium in any of them.
} 
current configuration of $\mathcal{B}$, and let $\theta_{0}$ be a uniform temperature field in the reference configuration. The motion and temperature history of $\mathcal{B}$ are then described by the two functions

$$
\mathbf{x}=\chi(\mathbf{X}, t), \quad \theta=\Theta(\mathbf{X}, t)
$$

These and all other functions are assumed to be as smooth as needed. The pair $\{\chi, \Theta\}$ is called a process. Let

$$
\begin{aligned}
\mathbf{v} & =\frac{D \mathbf{x}}{D t}=\dot{\mathbf{x}}=\frac{\partial \chi}{\partial t}, \quad \mathbf{F}=\frac{\partial \chi}{\partial \mathbf{X}}, \quad J=\operatorname{det} \mathbf{F}(>0), \\
\mathbf{g}_{0} & =\frac{\partial \Theta}{\partial \mathbf{X}}, \quad \mathbf{E}=\frac{1}{2}\left(\mathbf{F}^{T} \mathbf{F}-\mathbf{I}\right) .
\end{aligned}
$$

Here, $\mathbf{F}^{T}$ denotes the transpose of $\mathbf{F}, \mathbf{I}$ is the identity tensor, and the symmetric tensor $\mathbf{E}$ is the Lagrangian strain tensor. When the velocity and temperature fields are represented by their spatial descriptions, we may define their gradients $\mathbf{L}$ and $\mathbf{g}$ :

$$
\mathbf{L}=\frac{\partial \mathbf{v}}{\partial \mathbf{x}}=\dot{\mathbf{F}} \mathbf{F}^{-1}, \quad \mathbf{g}=\frac{\partial \theta}{\partial \mathbf{x}}=\mathbf{F}^{-T} \mathbf{g}_{0} .
$$

We recall that

$$
\dot{\mathbf{E}}=\mathbf{F}^{T} \mathbf{D F}
$$

where $\mathbf{D}=\frac{1}{2}\left(\mathbf{L}+\mathbf{L}^{T}\right)$ is the rate of deformation tensor.

The pair $(\mathbf{E}, \theta)$ may be regarded as a point in a 7-dimensional Euclidean straintemperature space $\mathcal{E}$, equipped with an inner product

$$
\left\langle\left(\mathbf{E}_{1}, \theta_{1}\right),\left(\mathbf{E}_{2}, \theta_{2}\right)\right\rangle=\mathbf{E}_{1} \cdot \mathbf{E}_{2}+\theta_{1} \theta_{2},
$$

where $\mathbf{A} \cdot \mathbf{B}=\operatorname{tr}\left(\mathbf{A}^{T} \mathbf{B}\right)$ is the usual inner product between two tensors $\mathbf{A}$ and $\mathbf{B}$. If the body $\mathcal{B}$ is composed of unconstrained material, then it can undergo an arbitrary process, and, for each particle $X \in \mathcal{B},(\mathbf{E}(\mathbf{X}, t), \theta(\mathbf{X}, t))$ will describe a general curve $\mathcal{C}$ in the 7 -dimensional region of $\mathcal{E}$ for which $\operatorname{det}(2 \mathbf{E}+\mathbf{I})>0$. Let this curve be parameterized by $\xi=\xi(t)$, with $\dot{\xi}>0$. We then have an inverse function $t=\hat{t}(\xi)$. In the presence of an internal constraint, $(\mathbf{E}(\mathbf{X}, t), \theta(\mathbf{X}, t))$ will be confined to a 6-dimensional Riemannian manifold embedded in $\mathcal{E}$, as will be discussed in Sect. 3.

For any process $\{\chi, \Theta\}$, we may define an associated process $\left\{\chi^{+}, \Theta^{+}\right\}$by

$$
\begin{aligned}
\chi^{+}\left(\mathbf{X}, t^{+}\right) & =\mathbf{Q}(t) \chi(\mathbf{X}, t)+\mathbf{c}(t), \quad t^{+}=t+a, \\
\Theta^{+}\left(\mathbf{X}, t^{+}\right) & =\Theta(\mathbf{X}, t),
\end{aligned}
$$

where $\mathbf{Q}(t)$ is a proper orthogonal tensor-valued function, $\mathbf{c}(t)$ is a vector-valued function, and $a$ is a constant. These processes correspond to superimposing rigid rotations $\mathbf{Q}$ and translations $\mathbf{c}$ on $\chi$, keeping the temperature the same, and shifting the origin of the timecoordinate. Under the transformations $(6)_{1,2,3}$, the fields $\mathbf{F}, J, \mathbf{E}, \mathbf{g}$, and $\mathbf{g}_{0}$ transform as

$$
\mathbf{F}^{+}=\mathbf{Q}(t) \mathbf{F}, \quad J^{+}=J, \quad \mathbf{E}^{+}=\mathbf{E}, \quad \frac{D \mathbf{E}^{+}}{D t^{+}}=\dot{\mathbf{E}}, \quad \mathbf{g}^{+}=\mathbf{Q}(t) \mathbf{g}, \quad \mathbf{g}_{0}^{+}=\mathbf{g}_{0} .
$$


Let $\rho_{0}(>0)$ and $\rho$ be the mass densities of $\mathcal{B}$ in its reference and current configurations, respectively. Conservation of mass yields

$$
\rho_{0}=\rho J
$$

and it is obvious that under the transformations $(6)_{1,2,3}$,

$$
\rho^{+}=\rho
$$

Let $\mathbf{b}$ be the body force per unit mass and denote the Cauchy stress tensor by $\mathbf{T}$. The pointwise equations for balance of linear and angular momentum are Cauchy's two laws

$$
\operatorname{div} \mathbf{T}+\rho \mathbf{b}=\rho \dot{\mathbf{v}}, \quad \mathbf{T}^{T}=\mathbf{T} .
$$

For internally unconstrained materials, it is assumed that if a material can experience a process $\{\chi, \Theta\}$, then it can also experience all the processes in (6) and that ${ }^{3}$

$$
\mathbf{T}^{+}=\mathbf{Q}(t) \mathbf{T} \mathbf{Q}^{T}(t) .
$$

It then follows from (10) 1 and (9) that $\dot{\mathbf{v}}^{+}-\mathbf{b}^{+}=\mathbf{Q}(t)(\dot{\mathbf{v}}-\mathbf{b})$.

The symmetric Piola-Kirchhoff stress tensor $\mathbf{S}$ is given by

$$
J \mathbf{T}=\mathbf{F S F}^{T}
$$

From (7) $)_{1,2},(11)$, and (12), it follows that $\mathbf{S}^{+}=\mathbf{S}$.

Let $\varepsilon$ be the internal energy per unit mass, and let $r$ be the heat supplied per unit mass per unit time. Also, let $\mathbf{q}$ and $\mathbf{q}_{0}$ denote the spatial and referential descriptions of the heat flux vector, so that

$$
J \mathbf{q}=\mathbf{F} \mathbf{q}_{0} .
$$

The field equation representing energy balance is given in spatial form by

$$
\rho \dot{\varepsilon}=\rho r-\operatorname{div} \mathbf{q}+\mathbf{T} \cdot \mathbf{D},
$$

and in referential form by

$$
\rho_{0} \dot{\varepsilon}=\rho_{0} r-\operatorname{Div} \mathbf{q}_{0}+\mathbf{S} \cdot \dot{\mathbf{E}} .
$$

In the absence of internal constraints, it is assumed that under the transformations $(6)_{1,2,3}$, $\varepsilon$ and $\mathbf{q}$ become

$$
\varepsilon^{+}=\varepsilon, \quad \mathbf{q}^{+}=\mathbf{Q}(t) \mathbf{q}
$$

and then $\mathbf{q}_{0}^{+}=\mathbf{q}_{0}$. Also, for internally unconstrained materials, it is clear from (9), (16) 1,2 , and (14) that $r^{+}=r$.

\footnotetext{
${ }^{3}$ For internally constrained materials, the "objectivity" condition (11) must be modified [12].
} 


\section{Unconstrained Thermoelastic Materials with Viscosity}

Consider a set $\mathcal{M}$ of materials defined by the constitutive relations

$$
\varepsilon=\tilde{\varepsilon}(\mathbf{F}, \theta), \quad \mathbf{T}=\tilde{\mathbf{T}}(\mathbf{F}, \dot{\mathbf{F}}, \theta, \mathbf{g}), \quad \mathbf{q}=\tilde{\mathbf{q}}(\mathbf{F}, \dot{\mathbf{F}}, \theta, \mathbf{g})
$$

We may set $\tilde{\varepsilon}\left(\mathbf{I}, \theta_{0}\right)=0$. We assume that when the temperature gradient vanishes, there is no heat flux:

$$
\tilde{\mathbf{q}}(\mathbf{F}, \dot{\mathbf{F}}, \theta, \mathbf{0})=\mathbf{0} .
$$

An element of the set $\mathcal{M}$ will be denoted by $m$. The form of the response functions in (17) $)_{1,2,3}$ depend on the reference configuration. Also, for inhomogeneous materials, the variable $\mathbf{X}$ may be understood to be added to the list of independent variables, in which case they may be said to define a material point.

Using standard arguments, it may be shown that the invariance requirements (11) and $(16)_{1,2}$ imply that $(17)_{1,2,3}$ can be expressed in the properly invariant form

$$
\varepsilon=\hat{\varepsilon}(\mathbf{E}, \theta), \quad \mathbf{S}=\hat{\mathbf{S}}\left(\mathbf{E}, \dot{\mathbf{E}}, \theta, \mathbf{g}_{0}\right), \quad \mathbf{q}_{0}=\hat{\mathbf{q}}_{0}\left(\mathbf{E}, \dot{\mathbf{E}}, \theta, \mathbf{g}_{0}\right) .
$$

Also $\hat{\varepsilon}\left(\mathbf{0}, \theta_{0}\right)=0$.

It follows from $(3)_{2},(13),(17)_{3},(19)_{3}$, and (18) that

$$
\hat{\mathbf{q}}_{0}(\mathbf{E}, \dot{\mathbf{E}}, \theta, \mathbf{0})=\mathbf{0} .
$$

As mentioned in the Introduction, a state of a particle $X$ satisfying the constitutive equations $(19)_{1,2,3}$ is uniquely determined by the value of the pair $(\mathbf{E}, \theta)$. Thus, each point in strain-temperature space $\mathcal{E}$ represents a unique state of the material point. ${ }^{4}$

The constitutive equations (17) $1,2,3$ hold for all processes, and in particular, for equilibrium processes, for which $\mathbf{F}$ is independent of time and $\theta$ is constant, and the state of $X$ is then fixed. Let

$$
\mathbf{T}^{e}=\mathbf{T}^{e}(\mathbf{F}, \theta)=\hat{\mathbf{T}}(\mathbf{F}, \mathbf{0}, \theta, \mathbf{0}),
$$

i.e., the restriction of $\hat{\mathbf{T}}$ gotten by setting $\dot{\mathbf{F}}$ and $\mathbf{g}$ equal to zero. If the material were held in equilibrium at the values $\mathbf{F}$ and $\theta$, the value $\mathbf{T}^{e}$ of Cauchy stress would be measured. Equation (21) is an equation of state associated with the constitutive equation $(17)_{2}$. Without any loss in generality, we may rewrite $(17)_{2}$ as

$$
\mathbf{T}=\mathbf{T}^{e}+\mathbf{T}^{*}(\mathbf{F}, \dot{\mathbf{F}}, \theta, \mathbf{g}),
$$

with

$$
\mathbf{T}^{*}(\mathbf{F}, \mathbf{0}, \theta, \mathbf{0})=\mathbf{0} .
$$

\footnotetext{
${ }^{4}$ In classical parlance, the variables $\mathbf{E}$ and $\theta$ are independent parameters of state. The dependent parameters of state, such as $\varepsilon$, can be expressed as functions of the independent parameters, yielding equations of state. The independent variables $\mathbf{F}$ and $\theta$, with which we started out in (17) 1 , also determine a unique state. However, the invariance requirements led us to the reduced set of independent variables $\mathbf{E}$ and $\theta$, with dimensionality 7 instead of 10. The variables $\dot{\mathbf{E}}$ and $\mathbf{g}_{0}$ are not parameters of state, but can influence $\mathbf{S}$ and $\mathbf{q}_{0}$, as indicated in $(19)_{2,3}$.
} 
Similarly,

$$
\mathbf{S}=\mathbf{S}^{e}(\mathbf{E}, \theta)+\mathbf{S}^{*}\left(\mathbf{E}, \dot{\mathbf{E}}, \theta, \mathbf{g}_{0}\right)
$$

with

$$
\mathbf{S}^{e}(\mathbf{E}, \theta)=\hat{\mathbf{S}}(\mathbf{E}, \mathbf{0}, \theta, \mathbf{0}), \quad J \mathbf{T}^{e}=\mathbf{F} \mathbf{S}^{e} \mathbf{F}^{T}, \quad J \mathbf{T}^{*}=\mathbf{F S}^{*} \mathbf{F}^{T}, \quad \mathbf{S}^{*}(\mathbf{E}, \mathbf{0}, \theta, \mathbf{0})=0 .
$$

It is important to observe that for non-equilibrium processes, the state of $X$ may change with time in an arbitrary fashion, but nonetheless, the values of the functions $\hat{\varepsilon}(\mathbf{E}, \theta)$ and $\mathbf{S}^{e}(\mathbf{E}, \theta)$ are determined by the instantaneous values of $\mathbf{E}$ and $\theta$.

A homothermal process is one for which the temperature is independent of position $\mathbf{x}$, and hence $\mathbf{g}=\mathbf{0}$, and equivalently, by $(3)_{2}, \mathbf{g}_{0}=\mathbf{0}$. By virtue of (20), for any homothermal process, the energy equation (15) reduces to

$$
\rho_{0} \dot{\varepsilon}=\rho_{0} r_{H}+\mathbf{S} \cdot \dot{\mathbf{E}}
$$

where $r_{H}$ signifies the heating that is associated with the homothermal process.

Employing the parameter $\xi(\dot{\xi}>0)$ for the curve $\mathcal{C}$ traced out by $(\mathbf{E}(t), \theta(t))$ in straintemperature space $\mathcal{E}$, we may express (26) as

$$
\rho_{0} \frac{D \varepsilon}{D \xi} \dot{\xi}=\rho_{0} r_{H}+\mathbf{S} \cdot \frac{D \mathbf{E}}{D \xi} \dot{\xi}
$$

Let

$$
\bar{r}_{H}=\frac{r_{H}}{\dot{\xi}} .
$$

Then, (27) becomes

$$
\rho_{0} \frac{D \varepsilon}{D \xi}=\rho_{0} \bar{r}_{H}+\mathbf{S} \cdot \frac{D \mathbf{E}}{D \xi} .
$$

Inserting (24) in (29), we obtain

$$
\rho_{0} \frac{D \varepsilon}{D \xi}=\rho_{0} \bar{r}_{H}+\left\{\mathbf{S}^{e}(\mathbf{E}, \theta)+\mathbf{S}^{*}\left(\mathbf{E}, \dot{\xi} \frac{D \mathbf{E}}{D \xi}, \theta, \mathbf{0}\right)\right\} \cdot \frac{D \mathbf{E}}{D \xi},
$$

which holds for every homothermal process.

We now consider a family of homothermal processes, such that for each $X \in \mathcal{B}$, $(\mathbf{E}(\mathbf{X}, t), \theta(\mathbf{X}, t))$ generates the same oriented curve $\mathcal{C}$ in strain-temperature space, but traverses it more and more slowly. In the limit as $\dot{\xi} \rightarrow 0$, (30) becomes

$$
\rho_{0} \frac{D \varepsilon}{D \xi}=\rho_{0} \bar{r}_{H 0}+\mathbf{S}^{e}(\mathbf{E}, \theta) \cdot \frac{D \mathbf{E}}{D \xi},
$$

where $(25)_{4}$ has been invoked and

$$
\bar{r}_{H 0}=\lim _{\dot{\xi} \rightarrow 0} \bar{r}_{H},
$$

which is assumed to exist. We call the limit of the family of ever more slow homothermal processes a "quasistatic homothermal process". 
"Part 1" of the second law of thermodynamics may be regarded as asserting that the Clausius integral

$$
I=\int_{\xi_{1}}^{\xi_{2}} \frac{\bar{r}_{H 0}}{\theta} d \xi
$$

is path-independent in strain-temperature space $\mathcal{E}$. Path-independence leads immediately to the existence of an entropy function $\eta=\hat{\eta}(\mathbf{E}, \theta)$, defined by

$$
\eta=\int_{\xi_{0}}^{\xi} \frac{\bar{r}_{H 0}}{\theta}(\alpha) d \alpha+\text { const. }
$$

where any path may be chosen between an arbitrary point $\left(\mathbf{E}\left(\xi_{0}\right), \theta\left(\xi_{0}\right)\right)$ and $(\mathbf{E}(\xi), \theta(\xi))$. We may choose the arbitrary constant in (34) such that $\hat{\eta}\left(\mathbf{0}, \theta_{0}\right)=0$.

It follows from (34) that for every quasistatic homothermal process

$$
\frac{D \eta}{D \xi}=\frac{\bar{r}_{H 0}}{\theta} \text {. }
$$

The Helmholtz free energy function $\psi=\hat{\psi}(\mathbf{E}, \theta)$ is given by

$$
\psi=\varepsilon-\eta \theta
$$

Note that $\hat{\psi}\left(\mathbf{0}, \theta_{0}\right)=0$. Clearly,

$$
\frac{D \psi}{D \xi}=\frac{D \varepsilon}{D \xi}-\frac{D \eta}{D \xi} \theta-\eta \frac{D \theta}{D \xi} .
$$

It follows from (31), (35), and (37) that for quasistatic homothermal processes

$$
\rho_{0} \frac{D \psi}{D \xi}=\mathbf{S}^{e}(\mathbf{E}, \theta) \cdot \frac{D \mathbf{E}}{D \xi}-\rho_{0} \eta \frac{D \theta}{D \xi} .
$$

Hence,

$$
\left\{\rho_{0} \frac{\partial \hat{\psi}}{\partial \mathbf{E}}-\mathbf{S}^{e}(\mathbf{E}, \theta)\right\} \cdot \frac{D \mathbf{E}}{D \xi}+\rho_{0}\left\{\frac{\partial \hat{\psi}}{\partial \theta}+\hat{\eta}(\mathbf{E}, \theta)\right\} \cdot \frac{D \theta}{D \xi}=0
$$

for all quasistatic homothermal processes. ${ }^{5}$ Since the terms in parentheses are independent of $\frac{D \mathbf{E}}{D \xi}$ and $\frac{D \theta}{D \xi}$, it follows that

$$
\mathbf{S}^{e}(\mathbf{E}, \theta)=\rho_{0} \frac{\partial \hat{\psi}}{\partial \mathbf{E}}(\mathbf{E}, \theta), \quad \hat{\eta}(\mathbf{E}, \theta)=-\frac{\partial \hat{\psi}}{\partial \theta}(\mathbf{E}, \theta) .
$$

Since each term in $(40)_{1}$ and $(40)_{2}$ is independent of both time rates and the gradient $\mathbf{g}_{0}$, these equations hold for all processes. They are called the Gibbs relations. Note that only the rate-independent part of the stress $\mathbf{S}$ is derivable from the Helmholtz free energy function.

By virtue of (40), for any process, we have

$$
\rho_{0} \dot{\psi}=\mathbf{S}^{e}(\mathbf{E}, \theta) \cdot \dot{\mathbf{E}}-\rho_{0} \hat{\eta}(\mathbf{E}, \theta) \dot{\theta}
$$

\footnotetext{
${ }^{5}$ The notation " $\frac{\partial \hat{\psi}}{\partial \mathbf{E}}$ " is used as an abbreviation for the symmetric tensor $\frac{1}{2}\left\{\frac{\partial \hat{\psi}}{\partial \mathbf{E}}+\left(\frac{\partial \hat{\psi}}{\partial \mathbf{E}}\right)^{T}\right\}$.
} 
This is the Gibbs equation. Equivalently, in view of (36),

$$
\rho_{0} \dot{\varepsilon}=\mathbf{S}^{e}(\mathbf{E}, \theta) \cdot \dot{\mathbf{E}}+\rho_{0} \theta \dot{\eta},
$$

which again holds for all processes.

The Gibbs relation (40) $)_{1}$ for $\mathbf{S}^{e}$ yields

$$
\mathbf{T}^{e}(\mathbf{F}, \theta)=\rho \mathbf{F} \frac{\partial \hat{\psi}}{\partial \mathbf{E}} \mathbf{F}^{T},
$$

where (8) and (25) 2 have been invoked. Similarly, (41) and (42) furnish

$$
\rho \dot{\psi}=\mathbf{T}^{e}(\mathbf{F}, \theta) \cdot \mathbf{D}-\rho \eta \dot{\theta}
$$

and

$$
\rho \dot{\varepsilon}=\mathbf{T}^{e}(\mathbf{F}, \theta) \cdot \mathbf{D}+\rho \theta \dot{\eta},
$$

where (4) has also been employed. ${ }^{6}$

With the help of (45) and (22), the energy equation (14) may be expressed in the form

$$
\rho \dot{\eta} \theta=\rho r-\operatorname{div} \mathbf{q}+\mathbf{T}^{*}(\mathbf{F}, \dot{\mathbf{F}}, \theta, \mathbf{g}) \cdot \mathbf{D} .
$$

Similarly, (15) may be expressed as

$$
\rho_{0} \dot{\eta} \theta=\rho_{0} r-\operatorname{Div} \mathbf{q}_{0}+\mathbf{S}^{*}\left(\mathbf{E}, \dot{\mathbf{E}}, \theta, \mathbf{g}_{0}\right) \cdot \dot{\mathbf{E}},
$$

where use has been made of (42) and (24).

We observe that a material $m \in \mathcal{M}$ may now be specified by providing constitutive equations for the functions $\hat{\psi}(\mathbf{E}, \theta), \mathbf{S}^{*}\left(\mathbf{E}, \dot{\mathbf{E}}, \theta, \mathbf{g}_{0}\right)$, and $\hat{\mathbf{q}}_{0}\left(\mathbf{E}, \dot{\mathbf{E}}, \theta, \mathbf{g}_{0}\right)$. The functions $\mathbf{S}^{e}(\mathbf{E}, \theta)$ and $\eta(\mathbf{E}, \theta)$ can then be obtained from the Gibbs relations $(40)_{1,2}$, while $\hat{\mathbf{S}}\left(\mathbf{E}, \dot{\mathbf{E}}, \theta, \mathbf{g}_{0}\right)$ can be found from (24) and (19) $)_{2}$ and $\hat{\varepsilon}(\mathbf{E}, \theta)$ can be obtained from (36).

As "Part 2" of the second law of thermodynamics, one may adopt any appropriate entropy inequality. For example, the Clausius-Duhem inequality implies that $[1,2]$

$$
\rho \dot{\eta} \theta \geq \rho r-\operatorname{div} \mathbf{q}+\frac{\mathbf{q} \cdot \mathbf{g}}{\theta}
$$

for all processes $\{\chi, \Theta\}$, the functions $\eta$ and $\mathbf{q}$ being provided by $(40)_{2}$ and $(17)_{3}$. The inequality (48) will place restrictions on the response functions, and hence on which members of $\mathcal{M}$ are thermodynamically allowable. For the class of materials being considered here, it follows from (46) and (48) that

$$
\mathbf{T}^{*}(\mathbf{F}, \dot{\mathbf{F}}, \theta, \mathbf{g}) \cdot \mathbf{D}-\frac{\mathbf{q} \cdot \mathbf{g}}{\theta} \geq 0 .
$$

For homothermal processes, (49) becomes

$$
\mathbf{T}^{*}(\mathbf{F}, \dot{\mathbf{F}}, \theta, \mathbf{0}) \cdot \mathbf{D} \geq 0 .
$$

\footnotetext{
${ }^{6}$ It is worth observing that in view of (45), entropy changes can be expressed in terms of changes in measurable quantities. 
If $\mathbf{T}^{*}$ were independent of $\mathbf{g}$, an inequality of the form (50) would hold for all processes. If we set $\dot{\mathbf{F}}=\mathbf{0}$ in (49), we obtain

$$
\tilde{\mathbf{q}}(\mathbf{F}, \mathbf{0}, \theta, \mathbf{g}) \cdot \mathbf{g} \leq 0 .
$$

If $\tilde{\mathbf{q}}$ were independent of $\dot{\mathbf{F}}$, an inequality of the form (51) would hold for all processes.

Remark 1 The class of thermoelastic materials is obtained from $(17)_{1,2,3}$ by taking the response functions $\tilde{\mathbf{T}}$ and $\tilde{\mathbf{q}}$ to be independent of the rate $\dot{\mathbf{F}}$. The Cauchy stress tensor $\mathbf{T}$ then coincides with $\mathbf{T}^{e}$, defined by (21). For thermoelastic materials, the Gibbs relation (43) holds for $\mathbf{T}$. Likewise, (40) 1 holds for $\mathbf{S}$. The rate-dependent variables in (46), (47), and (49) disappear. For more details, see [7, 10, 11].

\section{Strain-Temperature Internal Constraints}

Consider an internal constraint of the form

$$
\tilde{\phi}(\mathbf{F}, \theta)=0
$$

where $\tilde{\phi}$ is a sufficiently smooth scalar-valued function, with $\tilde{\phi}\left(\mathbf{I}, \theta_{0}\right)=0$. If (52) is satisfied by a process $\{\chi, \Theta\}$, we assume that it is also satisfied by the processes $\left\{\chi^{+}, \Theta^{+}\right\}$defined in $(6)_{1,2,3}$. It then follows that (52) can be expressed in the properly invariant form

$$
\phi(\mathbf{E}, \theta)=0,
$$

with $\phi\left(\mathbf{0}, \theta_{0}\right)=0$. Note that the internal constraint is described by a function $\phi$ of state. We assume that $\left(\frac{\partial \phi}{\partial \mathbf{E}}, \frac{\partial \phi}{\partial \theta}\right) \neq 0$, so that (53) defines a 6-dimensional hypersurface $\mathcal{S}$ embedded in the strain-temperature space $\mathcal{E}$. $\mathcal{S}$ is called the constraint manifold. In general, its geometry is Riemannian.

For any process satisfying (53), $(\mathbf{E}(t), \theta(t))$ will trace out a curve lying on $\mathcal{S}$, and

$$
\frac{\partial \phi}{\partial \mathbf{E}} \cdot \dot{\mathbf{E}}+\frac{\partial \phi}{\partial \theta} \dot{\theta}=0
$$

The vector $(\dot{\mathbf{E}}, \dot{\theta})$ lies in the tangent space $T_{P} \mathcal{S}$ to $\mathcal{S}$ at the point $P=(\mathbf{E}, \theta)$ and $\left(\frac{\partial \phi}{\partial \mathbf{E}}, \frac{\partial \phi}{\partial \theta}\right)$ is orthogonal to $\mathcal{S}$.

Following the procedure of Casey and Krishnaswamy [7], we utilize the constraint manifold $\mathcal{S}$ to induce an equivalence class structure on the set of unconstrained materials $\mathcal{M}$. Let $m_{1}$ and $m_{2}$ be any two materials in $\mathcal{M}$, specified as follows:

$$
\begin{array}{llll}
m_{1}: & \hat{\psi}_{1}(\mathbf{E}, \theta), & \mathbf{S}_{1}^{*}\left(\mathbf{E}, \dot{\mathbf{E}}, \theta, \mathbf{g}_{0}\right), & \hat{\mathbf{q}}_{01}\left(\mathbf{E}, \dot{\mathbf{E}}, \theta, \mathbf{g}_{0}\right), \\
m_{2}: & \hat{\psi}_{2}(\mathbf{E}, \theta), & \mathbf{S}_{2}^{*}\left(\mathbf{E}, \dot{\mathbf{E}}, \theta, \mathbf{g}_{0}\right), & \hat{\mathbf{q}}_{02}\left(\mathbf{E}, \dot{\mathbf{E}}, \theta, \mathbf{g}_{0}\right) .
\end{array}
$$

We may write

$$
\begin{aligned}
& \mathbf{S}_{1}=\mathbf{S}_{1}^{e}(\mathbf{E}, \theta)+\mathbf{S}_{1}^{*}\left(\mathbf{E}, \dot{\mathbf{E}}, \theta, \mathbf{g}_{0}\right)=\rho_{0} \frac{\partial \hat{\psi}_{1}}{\partial \mathbf{E}}(\mathbf{E}, \theta)+\mathbf{S}_{1}^{*}\left(\mathbf{E}, \dot{\mathbf{E}}, \theta, \mathbf{g}_{0}\right), \\
& \mathbf{S}_{2}=\mathbf{S}_{2}^{e}(\mathbf{E}, \theta)+\mathbf{S}_{2}^{*}\left(\mathbf{E}, \dot{\mathbf{E}}, \theta, \mathbf{g}_{0}\right)=\rho_{0} \frac{\partial \hat{\psi}_{2}}{\partial \mathbf{E}}(\mathbf{E}, \theta)+\mathbf{S}_{2}^{*}\left(\mathbf{E}, \dot{\mathbf{E}}, \theta, \mathbf{g}_{0}\right)
\end{aligned}
$$


and

$$
\hat{\eta}_{1}(\mathbf{E}, \theta)=-\frac{\partial \hat{\psi}_{1}}{\partial \theta}(\mathbf{E}, \theta), \quad \hat{\eta}_{2}(\mathbf{E}, \theta)=-\frac{\partial \hat{\psi}_{2}}{\partial \theta}(\mathbf{E}, \theta),
$$

where the Gibbs relations (40) $)_{1,2}$ have been employed for both $m_{1}$ and $m_{2}$.

The Helmholtz functions $\hat{\psi}_{1}(\mathbf{E}, \theta)$ and $\hat{\psi}_{2}(\mathbf{E}, \theta)$ of the unconstrained materials $m_{1}$ and $m_{2}$ can always be evaluated on the constraint manifold $\mathcal{S}$ and, in general, will not coincide with each other there. However, it is evident that for any given $\hat{\psi}_{1}(\mathbf{E}, \theta)$, there are infinitely many materials in $\mathcal{M}$ whose Helmholtz functions do coincide with $\hat{\psi}_{1}(\mathbf{E}, \theta)$ on $\mathcal{S}$. We may define an equivalence relation " $\sim$ " on $\mathcal{M}$ by $m_{1} \sim m_{2}$ if and only if ${ }^{7}$

$$
\begin{aligned}
\hat{\psi}_{1}(\mathbf{E}, \theta) & =\hat{\psi}_{2}(\mathbf{E}, \theta), \\
\mathbf{S}_{1}^{*}\left(\mathbf{E}, \dot{\mathbf{E}}, \theta, \mathbf{g}_{0}\right) & =\mathbf{S}_{2}^{*}\left(\mathbf{E}, \dot{\mathbf{E}}, \theta, \mathbf{g}_{0}\right), \\
\hat{\mathbf{q}}_{1}\left(\mathbf{E}, \dot{\mathbf{E}}, \theta, \mathbf{g}_{0}\right) & =\hat{\mathbf{q}}_{2}\left(\mathbf{E}, \dot{\mathbf{E}}, \theta, \mathbf{g}_{0}\right)
\end{aligned}
$$

for all $(\mathbf{E}, \theta) \in \mathcal{S}$, and for arbitrary values of $\dot{\mathbf{E}}$ and $\mathbf{g}_{0}$. When $m_{1} \sim m_{2}$, we say that $m_{1}$ "matches" $m_{2}$ on the constraint manifold $\mathcal{S}$. The equivalence class

$$
M(m)=\{n \in \mathcal{M}: n \sim m\}
$$

consists of all the materials in $\mathcal{M}$ that match a given material $m$ on $\mathcal{S}$. The equivalence relation " " partitions $\mathcal{M}$ into mutually disjoint nonempty equivalence classes. The set of equivalence classes, the quotient set, is denoted by $\mathcal{M} / \sim$.

Consider any two materials $m_{1}$ and $m_{2}$ that belong to the same equivalence class $M$. For each of these materials, the Gibbs equation (41) holds for all processes:

$$
\begin{aligned}
\rho_{0} \dot{\psi}_{1} & =\mathbf{S}_{1}^{e}(\mathbf{E}, \theta) \cdot \dot{\mathbf{E}}-\rho_{0} \hat{\eta}_{1}(\mathbf{E}, \theta) \dot{\theta}, \\
\rho_{0} \dot{\psi}_{2} & =\mathbf{S}_{2}^{e}(\mathbf{E}, \theta) \cdot \dot{\mathbf{E}}-\rho_{0} \hat{\eta}_{2}(\mathbf{E}, \theta) \dot{\theta} .
\end{aligned}
$$

Let both $m_{1}$ and $m_{2}$ undergo any process $\{\chi, \Theta\}$ that satisfies the constraint equation (53). Since these two materials match one another on $\mathcal{S}$, it follows from the condition (58) that

$$
\dot{\psi}_{2}=\dot{\psi}_{1} \quad(\text { on } \mathcal{S})
$$

for the process $\{\chi, \Theta\}$. In view of (61) and (60),

$$
\mathbf{S}_{2}^{e}(\mathbf{E}, \theta) \cdot \dot{\mathbf{E}}-\rho_{0} \hat{\eta}_{2}(\mathbf{E}, \theta) \dot{\theta}=\mathbf{S}_{1}^{e}(\mathbf{E}, \theta) \cdot \dot{\mathbf{E}}-\rho_{0} \hat{\eta}_{1}(\mathbf{E}, \theta) \dot{\theta}
$$

for all $\dot{\mathbf{E}}$ and $\dot{\theta}$ that satisfy (54). Introducing a Lagrange multiplier $\lambda(\mathbf{E}, \theta),(\mathbf{E}, \theta) \in \mathcal{S}$, we deduce from (62) and (54) that

$$
\left\{\mathbf{S}_{2}^{e}(\mathbf{E}, \theta)-\mathbf{S}_{1}^{e}(\mathbf{E}, \theta)-\lambda \frac{\partial \phi}{\partial \mathbf{E}}\right\} \cdot \dot{\mathbf{E}}+\left\{-\rho_{0} \hat{\eta}_{2}(\mathbf{E}, \theta)+\rho_{0} \hat{\eta}_{1}(\mathbf{E}, \theta)-\lambda \frac{\partial \phi}{\partial \theta}\right\} \dot{\theta}=0
$$

\footnotetext{
${ }^{7}$ Recall that an equivalence relation on a set is reflexive, symmetric, and transitive, i.e., $m \sim m, m \sim n$ implies
} that $n \sim m$, and $m \sim n$ and $m \sim p$ imply that $m \sim p$, for arbitrary elements $m, n, p$ of the set. 
for all $P \in \mathcal{S}$ and for all $(\dot{\mathbf{E}}, \dot{\theta})$ lying in the tangent space $T_{P} \mathcal{S}$. Since the terms in parentheses in (63) are independent of rates, we conclude that

$$
\mathbf{S}_{2}^{e}(\mathbf{E}, \theta)=\mathbf{S}_{1}^{e}(\mathbf{E}, \theta)+\lambda \frac{\partial \phi}{\partial \mathbf{E}}, \quad \rho_{0} \hat{\eta}_{2}(\mathbf{E}, \theta)=\rho_{0} \hat{\eta}_{1}(\mathbf{E}, \theta)-\lambda \frac{\partial \phi}{\partial \theta} \quad(\text { on } \mathcal{S})
$$

Thus, the quantity $\left(\mathbf{S}_{2}^{e}(\mathbf{E}, \theta),-\rho_{0} \hat{\eta}_{2}(\mathbf{E}, \theta)\right)$ evaluated on $\mathcal{S}$ for material $m_{2}$ is equal to $\left(\mathbf{S}_{1}^{e}(\mathbf{E}, \theta),-\rho_{0} \hat{\eta}_{1}(\mathbf{E}, \theta)\right)$ for material $m_{1}$ plus a component $\lambda\left(\frac{\partial \phi}{\partial \mathbf{E}}, \frac{\partial \phi}{\partial \theta}\right)$ which is orthogonal to $\mathcal{S}{ }^{8}$ If $m_{1}$ and $m_{2}$ are two specific materials in $M\left(m_{1}\right)$, then the function $\lambda(\mathbf{E}, \theta)$ in $(64)_{1,2}$ has a definite value at each point $P \in \mathcal{S}$.

Further, if (64) $)_{1,2}$ and (58) $)_{2,3}$ hold for any two materials $m_{1}, m_{2} \in \mathcal{M}$, then these materials must belong to the same equivalence class. For, if (64) $)_{1,2}$ hold for any $m_{1}, m_{2} \in \mathcal{M}$, then

$$
\left\{\mathbf{S}_{2}^{e}(\mathbf{E}, \theta)-\mathbf{S}_{1}^{e}(\mathbf{E}, \theta)\right\} \cdot \dot{\mathbf{E}}=\lambda \frac{\partial \phi}{\partial \mathbf{E}} \cdot \dot{\mathbf{E}}, \quad \rho_{0}\left\{-\hat{\eta}_{2}(\mathbf{E}, \theta)+\hat{\eta}_{1}(\mathbf{E}, \theta)\right\} \cdot \dot{\theta}=\lambda \frac{\partial \phi}{\partial \theta} \cdot \dot{\theta},
$$

for all $P \in \mathcal{S}$ and $(\dot{\mathbf{E}}, \dot{\theta}) \in T_{P} \mathcal{S}$. Adding $(65)_{1}$ to $(65)_{2}$, and taking note of (54), we deduce that (62) must hold. Hence, in view of the Gibbs equation (40), applied to each of the materials, the relation (61) must hold. Therefore,

$$
\hat{\psi}_{2}(\mathbf{E}, \theta)=\hat{\psi}_{1}(\mathbf{E}, \theta)+\text { const. } \quad(\text { on } \mathcal{S})
$$

But, $\hat{\psi}_{2}\left(\mathbf{0}, \theta_{0}\right)=0=\hat{\psi}_{1}\left(\mathbf{0}, \theta_{0}\right)$, and the constant must be zero. Therefore $(58)_{1}$ is satisfied. Since we are supposing that $(58)_{2,3}$ hold, it follows that $m_{2} \sim m_{1}$. In other words, it is not possible for $(64)_{1,2}$ to hold between materials that belong to distinct equivalence classes in $\mathcal{M} / \sim$.

For any two materials $m_{1}, m_{2}$ belonging to the same equivalence class $M$, it follows from $(64)_{1},(58)_{2}$, and $(56)_{1,2}$ that

$$
\mathbf{S}_{2}=\mathbf{S}_{1}+\lambda \frac{\partial \phi}{\partial \mathbf{E}}
$$

for all $(\mathbf{E}, \theta) \in \mathcal{S}$, and for arbitrary values of $\dot{\mathbf{E}}$ and $\mathbf{g}_{0}$. Correspondingly, in terms of Cauchy stress,

$$
\mathbf{T}_{2}=\mathbf{T}_{1}+\frac{\lambda}{J} \mathbf{F} \frac{\partial \phi}{\partial \mathbf{E}} \mathbf{F}^{T} \quad((\mathbf{E}, \theta) \in \mathcal{S})
$$

where (12) has been applied.

Using the partition $\mathcal{M} / \sim$ of the set $\mathcal{M}$ of unconstrained materials into equivalence classes, we now construct an associated set $\mathcal{M}^{\prime}$ of materials by creating a unique element $m^{\prime}$ for each equivalence class $M \in \mathcal{M} / \sim$, and by assigning distinct elements to distinct equivalence classes. This defines a one-to-one correspondence (i.e., a bijection) $\Phi$ between

\footnotetext{
${ }^{8}$ In general, $\left(\mathbf{S}_{1}^{e},-\rho_{0} \hat{\eta}_{1}\right)$ will have a component orthogonal to $\mathcal{S}$ as well as a component lying in the tangent space to $\mathcal{S}$.
} 
the sets $\mathcal{M} / \sim$ and $\mathcal{M}^{\prime}: m^{\prime}=\Phi(M), M=\Phi^{-1}\left(m^{\prime}\right)$. For each $m^{\prime} \in \mathcal{M}^{\prime}$, we assume that

(a) An internally constrained material $m^{\prime}$ can experience only processes that satisfy the strain-temperature constraint equation (53).

(b) Let an internally constrained material $m^{\prime}$ undergo any given process that satisfies the constraint (53). Let $m$ be any (unconstrained) material in the equivalence class $M=\Phi^{-1}\left(m^{\prime}\right)$, and let $m$ be subjected to the same process. Then, the values of $\mathbf{S}, \varepsilon$, and $\mathbf{q}_{0}$ that $m$ possesses during the process can also be experienced by $m^{\prime}$.

(c) If an internally constrained material $m^{\prime}$ can experience values $\mathbf{S}^{\prime}, \varepsilon^{\prime}$, and $\mathbf{q}_{0}^{\prime}$ in any given process, then there exists some (unconstrained) material $m \in M=\Phi^{-1}\left(m^{\prime}\right)$ that can take on these same values in the given process.

We proceed to investigate the properties of these newly defined internally constrained materials. Let $m^{\prime}$ be any member of $\mathcal{M}^{\prime}$ and let it undergo any process that satisfies the constraint equation (53). Suppose that $m^{\prime}$ experiences values $\mathbf{S}^{\prime}, \varepsilon^{\prime}$, and $\mathbf{q}_{0}^{\prime}$ in this process. Let $M=\Phi^{-1}\left(m^{\prime}\right)$ be the equivalence class corresponding to $m^{\prime}$. Then, by part (c) of (69), there exists some material $m_{1} \in M$ that can experience these same values. But, since $m_{1} \in$ $\mathcal{M}$, the values are given by constitutive equations. Thus,

$$
\mathbf{S}^{\prime}=\mathbf{S}_{1}\left(\mathbf{E}, \dot{\mathbf{E}}, \theta, \mathbf{g}_{0}\right), \quad \varepsilon^{\prime}=\hat{\varepsilon}_{1}(\mathbf{E}, \theta), \quad \mathbf{q}_{0}^{\prime}=\hat{\mathbf{q}}_{01}\left(\mathbf{E}, \dot{\mathbf{E}}, \theta, \mathbf{g}_{0}\right)
$$

with $(\mathbf{E}, \theta)$ restricted to $\mathcal{S}$. As shown in Sect. 3, $m_{1}$ possesses an entropy function, and the Gibbs relations $(40)_{1,2}$ are satisfied. We will assign the values

$$
\psi^{\prime}(\mathbf{E}, \theta)=\hat{\psi}_{1}(\mathbf{E}, \theta), \quad \eta^{\prime}=\hat{\eta}_{1}(\mathbf{E}, \theta) \quad(\text { on } \mathcal{S})
$$

to the constrained material $m^{\prime}$ for the given process.

Let $m_{2}$ be any other member of $M$, and let it undergo the same process. It will experience values $\mathbf{S}_{2}, \varepsilon_{2}, \mathbf{q}_{02}$ that are given by the constitutive equations for $m_{2}$. According to part (b) of (69), the constrained material $m^{\prime}$ can also experience the values $\mathbf{S}_{2}, \varepsilon_{2}, \mathbf{q}_{02}$. Thus, the values

$$
\overline{\mathbf{S}}^{\prime}=\mathbf{S}_{2}\left(\mathbf{E}, \dot{\mathbf{E}}, \theta, \mathbf{g}_{0}\right), \quad \bar{\varepsilon}^{\prime}=\hat{\varepsilon}_{2}(\mathbf{E}, \theta), \quad \overline{\mathbf{q}}_{0}^{\prime}=\hat{\mathbf{q}}_{02}\left(\mathbf{E}, \dot{\mathbf{E}}, \theta, \mathbf{g}_{0}\right),
$$

for $(\mathbf{E}, \theta) \in \mathcal{S}$ and arbitrary $\dot{\mathbf{E}}, \mathbf{g}_{0}$, are also possible values for $m^{\prime}$. We will assign the values

$$
\bar{\psi}^{\prime}(\mathbf{E}, \theta)=\hat{\psi}_{2}(\mathbf{E}, \theta), \quad \bar{\eta}^{\prime}=\hat{\eta}_{2}(\mathbf{E}, \theta) \quad(\text { on } \mathcal{S})
$$

to $m^{\prime}$ as also being possible.

Since $m_{1} \sim m_{2}$, it follows from $(58)_{1,2,3}$ that $\hat{\psi}_{1}, \mathbf{S}_{1}^{*}$, and $\hat{\mathbf{q}}_{1}$ coincide with $\hat{\psi}_{2}, \mathbf{S}_{2}^{*}$, and $\hat{\mathbf{q}}_{2}$, respectively, for $(\mathbf{E}, \theta) \in \mathcal{S}$ and arbitrary values of $\dot{\mathbf{E}}$ and $\mathbf{g}_{0}$. In particular, it is evident from $(58)_{1},(71)_{1}$, and $(73)_{1}$ that

$$
\bar{\psi}^{\prime}(\mathbf{E}, \theta)=\psi^{\prime}(\mathbf{E}, \theta) \quad(\text { on } \mathcal{S}) .
$$

Even though $m_{1} \sim m_{2}$, it is not the case, in general, that $\mathbf{S}_{1}^{e}(\mathbf{E}, \theta)$ coincides with $\mathbf{S}_{2}^{e}(\mathbf{E}, \theta)$ on $\mathcal{S}$. Instead, they are related by $(64)_{1}$. Similarly, $\mathbf{S}_{1}$ and $\mathbf{S}_{2}$ are related by (67). Further, the entropy functions $\hat{\eta}_{1}(\mathbf{E}, \theta)$ and $\hat{\eta}_{2}(\mathbf{E}, \theta)$ are related by $(64)_{2}$. 
It follows from $(70)_{1},(72)_{1},(67)_{1,2},(71)_{2},(73)_{2}$, and $(64)_{2}$ that, in any given process satisfying (53), the values of the symmetric Piola-Kirchhoff stress $\mathbf{S}$ and the entropy $\eta$ that a constrained material can experience are

$$
\mathbf{S}=\overline{\mathbf{S}}^{\prime}=\mathbf{S}_{1}\left(\mathbf{E}, \dot{\mathbf{E}}, \theta, \mathbf{g}_{0}\right)+\lambda \frac{\partial \phi}{\partial \mathbf{E}}, \quad \rho_{0} \eta=\rho_{0} \bar{\eta}^{\prime}=\rho_{0} \hat{\eta}_{1}(\mathbf{E}, \theta)-\lambda \frac{\partial \phi}{\partial \theta} \quad(\text { on } \mathcal{S}) .
$$

Thus, for a constrained material $m^{\prime}$, the pair $\left(\mathbf{S},-\rho_{0} \eta\right)$ has a determinate component which is obtained from the constitutive equation for any material $m_{1}$ belonging to the equivalence class $\Phi^{-1}\left(m^{\prime}\right)$ and a component $\lambda\left(\frac{\partial \phi}{\partial \mathbf{E}}, \frac{\partial \phi}{\partial \theta}\right)$ which is orthogonal to the tangent space $T_{P} \mathcal{S}$; the Lagrange multiplier $\lambda$ can have any of the infinitely many values that are permissible in (67) and (64) $)_{1,2}$, which hold for any two materials in the same equivalence class $\Phi^{-1}\left(\mathrm{~m}^{\prime}\right)$. In general, the determinate component does not lie just in $T_{P} \mathcal{S}$, but also has a component orthogonal to $T_{P} \mathcal{S}$.

Remark 2 We note that the sets $\mathcal{M}$ and $\mathcal{M}^{\prime}$, of unconstrained and internally constrained materials, have no members in common, since for all the materials in $\mathcal{M}$, the pair $\left(\mathbf{S},-\rho_{0} \eta\right)$ is fully determined by constitutive equations, whereas for every constrained material, there is an arbitrary component $\lambda\left(\frac{\partial \phi}{\partial \mathbf{E}}, \frac{\partial \phi}{\partial \theta}\right)$, which can be made non-vanishing by choosing $\lambda$ to be nonzero. Thus, $\mathcal{M} \cap \mathcal{M}^{\prime}=\emptyset$.

Remark 3 In equation $(75)_{1}, \mathbf{S}_{1}$ is the stress in a particular unconstrained material $m_{1}$, and obeys the objectivity requirement $\mathbf{S}_{1}^{+}=\mathbf{S}_{1}$ under the transformations (6) $)_{1,2,3}$. The constraint function $\phi$ is also objective. However, the essential arbitrariness of the Lagrange multiplier $\lambda$ precludes the assumption that $\lambda^{+}=\lambda$. We therefore cannot conclude that the stress $\mathbf{S}$ in an internally constrained material is unaltered under the transformations $(6)_{1,2,3} \cdot{ }^{9}$

Remark 4 Each internally constrained material $m^{\prime}$ is associated with a unique equivalence class $M=\Phi^{-1}\left(m^{\prime}\right)$ of unconstrained materials. By $(58)_{1}$, all of the materials in $M$ share the same values of Helmholtz free energy $\hat{\psi}(\mathbf{E}, \theta)$ on the 6-dimensional constraint manifold $\mathcal{S}$ embedded in 7-dimensional strain-temperature space $\mathcal{E}$. We have assigned this common value of $\hat{\psi}(\mathbf{E}, \theta),(\mathbf{E}, \theta) \in \mathcal{S}$, to the constrained material $m^{\prime}$. If we place 6 curvilinear coordinates $q^{\alpha}$ on $\mathcal{S}$ and take partial derivatives $\frac{\partial \psi}{\partial q^{\alpha}}, \alpha=1,2, \ldots, 6$, we can obtain a formula for the component of $\left(\mathbf{S},-\rho_{0} \eta\right)$ that lies in the tangent space $T_{P} \mathcal{S}$ (see Sect. 6 of [7]). ${ }^{10}$ However, $m^{\prime}$ always also possesses a component which is orthogonal to $T_{P} \mathcal{S}$. The representations $(75)_{1,2}$, which do not identify the tangential components of $\left(\mathbf{S},-\rho_{0} \eta\right)$ explicitly, are more convenient in practice.

Remark 5 A theory paralleling the present one may be developed for strain-entropy constraints of the form

$$
\phi^{*}(\mathbf{E}, \eta)=0
$$

\footnotetext{
${ }^{9}$ Casey and Carroll [12] offer the example of a rigid body, which is fully constrained internally. Under an arbitrary applied force system, an (ideal) rigid body undergoes a rigid motion. There is no constitutive equation for Cauchy stress $\mathbf{T} ; \mathbf{T}$ is entirely reactive. At rest, $\mathbf{T}$ may be zero, but in motion, it generally cannot be zero. If (11) 1 were to hold, and if $\mathbf{T}$ were zero at rest, it would have to vanish for all motions. Thus, we are led to a contradiction.

${ }^{10}$ For internally constrained hyperelastic materials, Carlson and Tortorelli [13] showed that the tangential component of stress is equal to the tangential derivative of the strain energy function.
} 
An equivalence relation $\sim^{*}$ is defined on the set $\mathcal{M}$ of unconstrained materials by replacing the Helmholtz free energy function $\hat{\psi}(\mathbf{E}, \theta)$ with the internal energy function $\hat{\varepsilon}(\mathbf{E}, \eta)$, evaluated on the strain-entropy constraint manifold. A new partition $\mathcal{M} / \sim^{*}$ is thereby obtained. Instead of (41), equation (42), written in terms of $\mathbf{E}$ and $\eta$, is employed to obtain expressions for $(\mathbf{S}, \theta)$ for materials belonging to the same equivalence class $M^{*} \in \mathcal{M} / \sim^{*}$. A set $\mathcal{M}^{*}$ of internally constrained materials is constructed by placing the members of $\mathcal{M}^{*}$ into one-to-one correspondence with the equivalence classes in $\mathcal{M} / \sim^{*}$.

For thermoelastic materials, this procedure is carried out in detail in [11]. Other types of internal constraints, such as stress-temperature constraints, have been considered in [14]. Different types of internally constrained materials exhibit interestingly different features and invite further investigation.

Open Access This article is distributed under the terms of the Creative Commons Attribution Noncommercial License which permits any noncommercial use, distribution, and reproduction in any medium, provided the original author(s) and source are credited.

\section{References}

1. Coleman, B.D., Noll, W.: The thermodynamics of elastic materials with heat conduction and viscosity. Arch. Ration. Mech. Anal. 13, 167-178 (1963)

2. Truesdell, C., Noll, W.: The non-linear field theories of mechanics. In: Flügge, S. (ed.) Handbuch der Physik, vol. III/1, pp. 1-579. Springer, Berlin (1965)

3. Carlson, D.E.: Linear thermoelasticity. In: Flügge, S. (ed.) Handbuch der Physik, vol. VIa/2, pp. 297345. Springer, Berlin (1972)

4. Rivlin, R.S.: Comments on some recent researches in thermodynamics. Recent Adv. Eng. Sci. 8, 1-23 (1973)

5. Rivlin, R.S.: The thermomechanics of materials with fading memory. In: Hutton, J.F., Pearson, J.R.A., Walters, K. (eds.) Theoretical Rheology, pp. 83-103. Applied Science Publishers, Barking (1975). Reprinted in: Barenblatt, G.L., Joseph, D. (eds.) Collected Papers, vol. 2, pp. 1559-1579. Springer, Berlin (1997)

6. Rivlin, R.S.: Reflections on certains aspects of thermomechanics. In: Contributi del Centro Linceo Interdisciplinare di Scienze Matemitiche e loro Applicazioni, vol. 76, pp. 11-43. Meeting on Finite Thermoelasticity, Rome, 30 May-1 June 1986. Reprinted in: Barenblatt, G.L., Joseph, D. (eds.) Collected Papers, vol. 1, pp. 430-463. Springer, Berlin (1997)

7. Casey, J., Krishnaswamy, S.: A characterization of internally constrained thermoelastic materials. Math. Mech. Solids 3, 71-89 (1998)

8. Casey, J.: On elastic-thermo-plastic materials at finite deformations. Int. J. Plast. 14, 173-191 (1998)

9. Krishnaswamy, S., Batra, R.C.: A thermodynamical theory of solid-fluid mixtures. Math. Mech. Solids 2, 143-151 (1997)

10. Casey, J.: A remark on Cauchy elasticity. Int. J. Non-Linear Mech. 40, 331-339 (2005)

11. Casey, J.: Strain-temperature and strain-entropy internal constraints in finite thermoelasticity. In: Casey, J., Abeyaratne, R. (eds.) Finite Thermoelasticity. AMD, vol. 236, pp. 45-65. The American Society of Mechanical Engineers, New York (1999)

12. Casey, J., Carroll, M.M.: Discussion of "A treatment of internally constrained materials". J. Appl. Mech. 63, 240 (1996)

13. Carlson, D.E., Tortorelli, D.A.: On hyperelasticity with internal constraints. J. Elast. 42, 91-98 (1996)

14. Rooney, F.J., Bechtel, S.E.: Constraints, constitutive limits, and instability in finite thermoelasticity. J. Elast. 74, 109-133 (2004) 